\title{
On the Relationship between Interval Observers and Invariant Sets in Fault Detection
}

\author{
Feng Xu, Florin Stoican, Vicenç Puig, Carlos Ocampo-Martinez and Sorin Olaru
}

\begin{abstract}
In this paper, the relationship between two settheoretic fault detection (FD) approaches, the interval observerbased and the invariant set-based approaches, is investigated. In FD, interval observers monitor the system dynamic behavior in real time and generates adaptive intervals for system outputs. Invariant sets focus more on steady state behavior of the system rather than the transient behavior. This paper discusses these two approaches, presents the relationship between them and compares them in the FD task. At the end, a numerical example is used to illustrate the relation between these two approaches.
\end{abstract}

\section{INTRODUCTION}

Interval observer-based fault detection (FD) consists in generating adaptive intervals for system outputs by considering the bounds of uncertainties, propagating their effect through the mathematical models of the system and testing the consistency between the predicted output intervals and the corresponding measurements of outputs [4]-[6], [9], [10].

Another set-theoretic fault detection and isolation (FDI) approach is to consider invariant sets. As known, a system can switch among several modes (a healthy one and at least a faulty one). For each mode, an invariant set for the residual can be obtained [8], [11]. Once the system operates in steady state, it is possible to confine the residual to one of these invariant sets and, as long as all the invariant sets are disjoint, FDI can be performed. Most importantly, in the case that the invariant sets intersect, FD can still be done whenever the residual exits its healthy invariant set [7], [8], [12], [13].

So far, the FI application of interval observers has been blank in the scientific community, so the discussions of this paper are restricted in FD. The main objective of this paper is to establish the relationship between these two FD approaches, analyze their relative strengths and weakness and explore the advantages of combining both approaches to implement more efficient fault diagnosis mechanisms.

The main contribution of this paper is twofold. First, it presents the relationship between the two FD approaches. Second, it analyzes and compares the relative advantages and disadvantages of both approaches. It is considered that both approaches have complementary roles during the transient and steady state of the system, respectively.

F. Xu, V. Puig and C. Ocampo-Martinez are with the Institut de Robòtica i Informàtica Industrial (CSIC-UPC), Technical University of Catalonia, Llorens i Artigas, 4-6, 08028 Barcelona, Spain, \{fxu, vpuig, cocampo\}@iri.upc.edu.

F. Stoican is with the Politehnica University of Bucharest (PUB) Department of Automatic Control and Systems Engineering, Bucharest, Romania, florin.stoican@acse.pub.ro.

S. Olaru is with the E3S (SUPELEC Systems Sceinces), Automatic Control Departement, Gif sur Yvette, France, sorin.olaru@supelec.fr.
Taking into account the balance among the expressional compactness, computational precision and complexity, this paper will use zonotopes to represent and propagate uncertainty in system states and outputs [1]-[3].

Section II introduces the notion of zonotopes and invariant sets. Section III reviews the mathematical formulations of the two set theoretic FD methods. The theoretical relationship between both approaches is derived in Section IV. In Section $\mathrm{V}$, the comparison of the two methods is discussed. In Section VI, a numerical example is used to comment the relationship. Section VII draws the conclusions.

\section{PREliminaries}

The notation $\oplus$ represents the Minkowski sum, |.| denotes the elementwise absolute value, $\mathbf{B}^{r}$ is a $r$-dimensional unitary box and the inequalities are interpreted elementwise.

\section{A. Zonotopes}

According to [1], [3] and [4], several definitions and properties related to zonotopes are introduced as follows.

Definition 2.1: The Minkowski sum of two sets $\mathrm{A}$ and $\mathrm{B}$ is defined by $A \oplus B=\{a+b: a \in A, b \in B\}$.

Definition 2.2: Given a vector $p \in \mathbb{R}^{n}$ and a matrix $G \in \mathbb{R}^{n \times m}(n \leq m)$, a zonotope $X$ with order $m$ is defined as $X=p \oplus G \mathbf{B}^{m}$, where $p$ and $G$ are called the center and segment matrix of the zonotope, respectively.

Definition 2.3: The interval hull $\square X$ of a zonotope $X=p \oplus G \mathbf{B}^{r} \subset \mathbb{R}^{n}$ is the smallest interval box that contains $X$, i.e., $\square X=\left\{x:\left|x_{i}-p_{i}\right| \leq\left\|G_{i}\right\|_{1}\right\}$, where $G_{i}$ is the $i$-th row of $G$, and $x_{i}$ and $p_{i}$ are the $i$-th components of $x$ and $p$, respectively.

Property 2.1: Given zonotopes $X_{1}=p_{1} \oplus G_{1} \mathbf{B}^{r_{1}} \subset \mathbb{R}^{n}$ and $X_{2}=p_{2} \oplus G_{2} \mathbf{B}^{r_{2}} \subset \mathbb{R}^{n}$, the Minkowski sum of them is $X_{1} \oplus X_{2}=\left\{p_{1}+p_{2}\right\} \oplus\left[\begin{array}{ll}G_{1} & G_{2}\end{array}\right] \mathbf{B}^{r_{1}+r_{2}}$.

Property 2.2: Given a zonotope $X=p \oplus G \mathbf{B}^{r} \subset \mathbb{R}^{n}$ and a compatible matrix $K, K X=K p \oplus K G \mathbf{B}^{r}$ holds.

Property 2.3: (see [3]) Given a zonotope $X=p \oplus G \mathbf{B}^{r} \subset$ $\mathbb{R}^{n}$ and an integer $s$ (with $n<s<r$ ), denote by $\hat{G}$ the matrix resulting from the recording of the columns of the matrix $G$ in decreasing Euclidean norm. $X \subseteq p \oplus\left[\hat{G}_{T} \quad Q\right] \mathbf{B}^{s}$ where $\hat{G}_{T}$ is obtained from the first $s-n$ columns of matrix $\hat{G}$ and $Q \in \mathbb{R}^{n \times n}$ is a diagonal matrix whose elements satisfy $Q_{i i}=\sum_{j=s-n+1}^{r}\left|\hat{G}_{i j}\right|, i=1, \ldots, n$.

\section{B. Invariant Sets}

The linear discrete time-invariant dynamics

$$
x_{k+1}=A_{\circ} x_{k}+B_{\circ} \delta_{k}
$$


is used to present the invariant set notions, where $A_{\circ}$ and $B_{\circ}$ are constant matrices and $A_{\circ}$ is a Schur matrix, $\delta_{k}$ belongs to $\Delta=\left\{\delta:\left|\delta-\delta^{\circ}\right| \leq \bar{\delta}\right\}$ with $\delta^{\circ}$ and $\bar{\delta}$ constant.

Definition 2.4: A set $X \subset \mathbb{R}^{n}$ is called a robust positively invariant (RPI) set for (1) if and only if $A_{\circ} X \oplus B_{\circ} \Delta \subseteq X$.

Definition 2.5: The minimal RPI ( $m R P I)$ set of (1) is defined as a $R P I$ set contained in any closed $R P I$ set and the $m R P I$ set is unique and compact.

Theorem 2.1: (see [8]) Considering (1) and letting $A_{\circ}=$ $V \Lambda V^{-1}$ be the Jordan decomposition of $A_{\circ}$,

$$
\begin{aligned}
\Phi(\theta)= & \left\{x \in \mathbb{R}^{n}:\left|V^{-1} x\right| \leq(I-|\Lambda|)^{-1}\left|V^{-1} B_{\circ}\right| \bar{\delta}\right. \\
& +\theta\} \oplus \xi^{\circ},
\end{aligned}
$$

is RPI and attractive for the trajectories of (1), with $\theta$ any (arbitrarily small) vector with positive components, where $\xi^{\circ}$ is the center of the set with $\xi^{\circ}=\left(I-A_{\circ}\right)^{-1} B_{\circ} \delta^{\circ}$, where $I$ is the compatible identity matrix.

1) For any $\theta$, the set $\Phi(\theta)$ is (positively) invariant, that is, if $x_{0} \in \Phi(\theta)$, then $x_{k} \in \Phi(\theta)$ for all $k \geq 0$.

2) Given $\theta \in \mathbb{R}^{n}, \theta>0$, and $x_{0} \in \mathbb{R}^{n}$, there exists $k^{*} \geq 0$ such that $x_{k} \in \Phi(\theta)$ for all $k \geq k^{*}$.

Proposition 2.1: (see [8]) Considering (1) and denoting $X_{0}$ as a RPI initial set of (1), each of the set iterations

$$
X_{j+1}=A_{\circ} X_{j} \oplus B_{\circ} \Delta, \quad j \in \mathbb{N},
$$

where $j$ denotes the $j$-th element of the set sequence and $\mathbb{N}$ represents the set of natural numbers, is a $R P I$ approximation of the $m R P I$ set. Furthermore, as $j$ tends to infinity, the set sequence converges to the $m R P I$ set.

\section{Set Theoretic Methods in FD}

\section{A. Dynamical Models}

The linear discrete time-invariant plant is modeled as

$$
\begin{aligned}
x_{k+1} & =A x_{k}+B u_{k}+E \omega_{k}, \\
y_{k} & =C x_{k}+\eta_{k},
\end{aligned}
$$

where $A \in \mathbb{R}^{n \times n}, B \in \mathbb{R}^{n \times p}, C \in \mathbb{R}^{q \times n}$ and $E \in \mathbb{R}^{n \times r}$ are constant matrices, $x_{k} \in \mathbb{R}^{n}, u_{k} \in \mathbb{R}^{p}$ and $y_{k} \in \mathbb{R}^{q}$ are states, inputs and outputs, respectively, $\omega_{k} \in W$ and $\eta_{k} \in V$ are bounded disturbances and noises, respectively, and $k$ denotes the $k$-th discrete time instant. $W$ and $V$ are defined by

$$
\begin{aligned}
W & =\left\{\omega_{k} \in \mathbb{R}^{r}:\left|\omega_{k}-\omega^{c}\right| \leq \bar{\omega}, \omega^{c} \in \mathbb{R}^{r}, \bar{\omega} \in \mathbb{R}^{r}\right\} \\
V & =\left\{\eta_{k} \in \mathbb{R}^{q}:\left|\eta_{k}-\eta^{c}\right| \leq \bar{\eta}, \eta^{c} \in \mathbb{R}^{q}, \bar{\eta} \in \mathbb{R}^{q}\right\}
\end{aligned}
$$

where $\omega^{c}, \eta^{c}, \bar{\omega}$ and $\bar{\eta}$ are constant vectors. Due to the structure shown in (3) and (4), the two sets are rewritten as two zonotopes

$$
\begin{aligned}
W & =\omega^{c} \oplus H_{\bar{\omega}} \mathbf{B}^{r}, \\
V & =\eta^{c} \oplus H_{\bar{\eta}} \mathbf{B}^{q},
\end{aligned}
$$

where $H_{\bar{\omega}} \in \mathbb{R}^{r \times r}$ and $H_{\bar{\eta}} \in \mathbb{R}^{q \times q}$ are two diagonal matrices with the diagonal entries from $\bar{\omega}$ and $\bar{\eta}$, respectively.

Remark 3.1: For the sake of discussional generality, the centers of $W$ and $V$ are not restricted to be at the origin. $\diamond$

\section{B. Interval Observer-based FD}

The plant (2) is monitored by a linear Luenberger interval observer. The set-based form of the interval observer, based on the nominal model of the plant, is designed as

$$
\begin{aligned}
\hat{X}_{k+1}= & (A-L C) \hat{X}_{k} \oplus\left\{B u_{k}\right\} \oplus\left\{L y_{k}\right\} \\
& \oplus(-L) V \oplus E W, \\
\hat{Y}_{k}= & C \hat{X}_{k} \oplus V,
\end{aligned}
$$

where $\hat{X}_{k}$ and $\hat{Y}_{k}$ are real-time predicted state and output zonotopes at time instant $k$, respectively.

Assumption 3.1: The gain matrix $L$ is chosen to assure the set-mapping contractiveness of the interval observer and the avoidance of the wrapping effect [6].

According to (7), the center $\hat{x}_{k+1}^{c}$ and segment matrix $\hat{H}_{k+1}^{x}$ of $\hat{X}_{k+1}$, and the center $\hat{y}_{k}^{c}$ and segment matrix $\hat{H}_{k}^{y}$ of $\hat{Y}_{k}$ are computed as

$$
\begin{aligned}
\hat{x}_{k+1}^{c} & =(A-L C) \hat{x}_{k}^{c}+B u_{k}+L y_{k}-L \eta^{c}+E w^{c}, \\
\hat{H}_{k+1}^{x} & =\left[\begin{array}{lll}
(A-L C) \hat{H}_{k}^{x} & -L H_{\bar{\eta}} & E H_{\bar{\omega}}
\end{array}\right], \\
\hat{y}_{k}^{c} & =C \hat{x}_{k}^{c}+\eta^{c}, \\
\hat{H}_{k}^{y} & =\left[\begin{array}{ll}
C \hat{H}_{k}^{x} & H_{\bar{\eta}}
\end{array}\right] .
\end{aligned}
$$

Assumption 3.2: The initial state of the plant is denoted as $x_{0}$ and $x_{0}$ belongs to the initial zonotope $\hat{X}_{0}=\hat{x}_{0}^{c} \oplus \hat{H}_{0} \mathbf{B}^{s_{0}}$ of the interval observer.

In (8), as $k$ increases, the order of segment matrices of zonotopes grows dramatically. Thus, Property 2.3 is used to reduce the order of $\hat{H}_{k+1}^{x}$. Besides, residual zonotopes of the interval observer-based FD approach are defined as

$$
\begin{aligned}
R_{k}^{i o} & =\left\{y_{k}\right\} \oplus\left(-\hat{Y}_{k}\right) \\
& =\left\{C x_{k}+\eta_{k}\right\} \oplus\left\{\left(-C \hat{X}_{k}\right) \oplus(-V)\right\} \\
& =C\left\{\left\{x_{k}\right\} \oplus\left(-\hat{X}_{k}\right)\right\} \oplus\left\{\eta_{k}\right\} \oplus(-V) .
\end{aligned}
$$

According to [6], the interval observer-based FD consists in checking if

$$
\mathbf{0} \in \square R_{k}^{i o}
$$

is violated, where $\mathbf{0}$ is the zero vector. Thus, if (10) is violated, it means that a fault occurs. Otherwise, it is considered that the system is still in the healthy functioning.

\section{Invariant Set-based FD}

According to the invariant set-based approach [12], [13], a Luenberger observer for (2) is designed as

$$
\begin{aligned}
\hat{x}_{k+1} & =A \hat{x}_{k}+B u_{k}+L\left(y_{k}-C \hat{x}_{k}\right), \\
\hat{y}_{k} & =C \hat{x}_{k} .
\end{aligned}
$$

and the residual for the invariant set-based FD is defined as

$$
\begin{aligned}
r_{k}^{i s} & =y_{k}-\hat{y}_{k} \\
& =C\left(x_{k}-\hat{x}_{k}\right)+\eta_{k},
\end{aligned}
$$

where $x_{k}-\hat{x}_{k}$ is the state estimation error notated as $\tilde{x}_{k}$. Considering (2) and (11), the dynamics of $\tilde{x}_{k}$ is derived as

$$
\tilde{x}_{k+1}=(A-L C) \tilde{x}_{k}-L \eta_{k}+E \omega_{k} .
$$


Remark 3.2: For comparative convenience, the observer gain of (11) is chosen to be equal to that of (7) and the observer contractiveness is assured by Assumption 3.1. $\downarrow$

According to Theorem 2.1 and Proposition 2.1, a RPI set is constructed for (13). The resultant RPI set is denoted as $\Phi^{\tilde{x}}$ and the corresponding residual set is computed as

$$
R^{i s}=C \Phi^{\tilde{x}} \oplus V \text {. }
$$

Whenever $\tilde{x}_{k}$ is inside $\Phi^{\tilde{x}}, r_{k}^{i s}$ is inside $R^{i s}$. Thus, the invariant set-based FD is to test whether the residual (12) is located inside its healthy set (14) in real time. If the residual exits its healthy invariant set, it indicates that a fault occurs. Otherwise, it is assumed that the system is still healthy.

Since the computation of interval vectors is based on the interval hull of zonotopes, in the sequel and for simplicity, all the discussions are directly based on zonotopes.

\section{TheOretical Relationship}

The relationship between the two FD approaches is derived in this section. To the best of the authors' knowledge, this type of analysis is not available in the literature.

\section{A. Bounds of Interval Observers}

In order to describe residual zonotopes (9), a zonotope

$$
\begin{aligned}
\tilde{X}_{k} & =\left\{x_{k}\right\} \oplus\left(-\hat{X}_{k}\right) \\
& =\left(x_{k}-\hat{x}_{k}^{c}\right) \oplus \hat{H}_{k}^{x} \mathbf{B}^{s_{k}}
\end{aligned}
$$

is defined, where $\hat{X}_{k}=\hat{x}_{k}^{c} \oplus \hat{H}_{k} \mathbf{B}^{s_{k}}$. By using $\tilde{x}_{k}^{c}$ and $\tilde{H}_{k}$ to characterize $x_{k}-\hat{x}_{k}^{c}$ and $\hat{H}_{k}^{x}$, respectively, $\tilde{X}_{k}$ is rewritten as $\tilde{X}_{k}=\tilde{x}_{k}^{c} \oplus \tilde{H}_{k} \mathbf{B}^{s_{k}}$. Taking into account (2a), (8a) and (8b), the center and segment matrix of $\tilde{X}_{k+1}$ are derived as

$$
\begin{aligned}
\tilde{x}_{k+1}^{c} & =(A-L C) \tilde{x}_{k}^{c}-L\left(\eta_{k}-\eta^{c}\right)+E\left(\omega_{k}-\omega^{c}\right), \\
\tilde{H}_{k+1} & =\hat{H}_{k+1}^{x}=\left[\begin{array}{lll}
(A-L C) \hat{H}_{k}^{x} & -L H_{\bar{\eta}} & E H_{\bar{\omega}}
\end{array}\right] .
\end{aligned}
$$

According to Property 2.1 and Property 2.2, an equivalent zonotope-based form of (16) is deduced as

$$
\begin{aligned}
\tilde{X}_{k+1}= & (A-L C) \tilde{X}_{k} \oplus(-L)\left[\left(\eta_{k}-\eta^{c}\right) \oplus H_{\bar{\eta}} \mathbf{B}^{q}\right] \\
& \oplus E\left[\left(\omega_{k}-\omega^{c}\right) \oplus H_{\bar{\omega}} \mathbf{B}^{r}\right] .
\end{aligned}
$$

According to (15), the left side of (17) is expressed as

$$
\begin{aligned}
\tilde{X}_{k+1} & =\tilde{x}_{k+1}^{c} \oplus \tilde{H}_{k+1} \mathbf{B}^{s_{k+1}} \\
& =\left(x_{k+1}-\hat{x}_{k+1}^{c}\right) \oplus \hat{H}_{k+1}^{x} \mathbf{B}^{s_{k+1}} \\
& =x_{k+1} \oplus\left[\left(-\hat{x}_{k+1}^{c}\right) \oplus \hat{H}_{k+1}^{x} \mathbf{B}^{s_{k+1}}\right],
\end{aligned}
$$

while the right side of (17) can be rewritten as

$$
\begin{aligned}
\tilde{X}_{k+1}= & (A-L C)\left[\left(x_{k}-\hat{x}_{k}^{c}\right) \oplus \hat{H}_{k}^{x} \mathbf{B}^{s_{k}}\right] \oplus(-L)\left[\left(\eta_{k}-\eta^{c}\right)\right. \\
& \left.\oplus H_{\bar{\eta}} \mathbf{B}^{q}\right] \oplus E\left[\left(\omega_{k}-\omega^{c}\right) \oplus H_{\bar{\omega}} \mathbf{B}^{r}\right] \\
= & (A-L C) x_{k} \oplus(A-L C)\left[\left(-\hat{x}_{k}^{c}\right) \oplus \hat{H}_{k}^{x} \mathbf{B}^{s_{k}}\right] \\
& \oplus(-L) \eta_{k} \oplus(-L)\left[\left(-\eta^{c}\right) \oplus H_{\bar{\eta}} \mathbf{B}^{q}\right] \\
& \oplus E \omega_{k} \oplus E\left[\left(-\omega^{c}\right) \oplus H_{\bar{\omega}} \mathbf{B}^{r}\right] .
\end{aligned}
$$

When (7) predicts state and output zonotopes, one only uses the bounds of disturbances and noises. Thus, one uses $\check{\omega}_{k} \in W$ and $\check{\eta}_{k} \in V$ to describe $W$ and $V$ appearing in (7), respectively. Thus, one has that (18) and (19) correspond to $x_{k+1}-\hat{x}_{k+1}$ and $(A-L C)\left(x_{k}-\hat{x}_{k}\right)-L \eta_{k}+E \omega_{k}+$ $L \check{\eta}_{k}-E \check{\omega}_{k}$, respectively. Finally, by using $\breve{x}_{k}$ to characterize $x_{k}-\hat{x}_{k}$, one obtains the corresponding dynamics of $(17)^{1}$

$$
\breve{x}_{k+1}=(A-L C) \breve{x}_{k}-L \eta_{k}+E \omega_{k}+L \check{\eta}_{k}-E \check{\omega}_{k} .
$$

Using $W$ and $V$ to replace $\check{\omega}_{k}$ and $\omega_{k}$, and $\check{\eta}_{k}$ and $\eta_{k}$ in (20), a set-based form of (20) is obtained as

$$
\breve{X}_{k+1}=(A-L C) \breve{X}_{k} \oplus(-L) V \oplus E W \oplus L V \oplus(-E) W
$$

According to (21) and zonotope operations, the center $\breve{x}_{k+1}^{c}$ and segment matrix $\breve{H}_{k+1}$ of $\breve{X}_{k+1}$ are derived as

$\breve{x}_{k+1}^{c}=(A-L C) \breve{x}_{k}^{c}$,

$\breve{H}_{k+1}=\left[\begin{array}{lllll}(A-L C) \breve{H}_{k} & -L H_{\bar{\eta}} & E H_{\bar{\omega}} & L H_{\bar{\eta}} & -E H_{\bar{\omega}}\end{array}\right]$.

By comparing (17) and (21), it is shown that zonotopes predicted by (21) bound those predicted by (17) at each time instant, as long as $\tilde{X}_{0} \subseteq \breve{X}_{0}$ holds.

Finally, according to (9) and (21), zonotopes bounding residual zonotopes are derived as

$$
\breve{R}_{k}^{i o}=C \breve{X}_{k} \oplus V \oplus(-V) .
$$

\section{B. Relationship in Terms of Residuals}

1) Relationship of Intermediate Sets: A RPI set of (13) can be constructed by Theorem 2.1, which is denoted as $\Phi_{0}^{\tilde{x}}$ with center $\xi_{0}^{c}$. By using $\Phi_{0}^{\tilde{x}}$ as an initial set according to Proposition 2.1, another squeezed RPI set with arbitrarily expected precision to the mRPI set of (13) is obtained by

$$
\Phi_{j+1}^{\tilde{x}}=(A-L C) \Phi_{j}^{\tilde{x}} \oplus(-L) V \oplus E W, \quad j \in \mathbb{N},
$$

where $j$ represents the $j$-th element of this set sequence.

Since $W$ and $V$ are zonotopes, the construction of $\Phi_{0}^{\tilde{x}}$ implies that it is also a zonotope. Thus, $\Phi_{j+1}^{\tilde{x}}$ in (24) can be unfolded into a similar form with (16), with center $\xi_{j+1}^{c}$ and segment matrix $H_{j+1}^{\tilde{x}}$, where

$$
\begin{aligned}
\xi_{j+1}^{c} & =(A-L C) \xi_{j}^{c}-L \eta^{c}+E \omega^{c}, \\
H_{j+1}^{\tilde{x}} & =\left[\begin{array}{lll}
(A-L C) H_{j}^{\tilde{x}} & -L H_{\bar{\eta}} & E H_{\bar{\omega}}
\end{array}\right] .
\end{aligned}
$$

According to Proposition 2.1, as $j$ tends to infinity, the set sequence (24) converges to the mRPI set of (13), denoted as $\Phi_{\infty}^{\tilde{x}}$ with center $\xi_{\infty}^{c}$. Furthermore, according to (16), (22) and (25), as $k$ and $j$ tend to infinity, one has

$$
\begin{aligned}
\tilde{x}_{\infty}^{c}= & {[I-(A-L C)]^{-1}\left[\left(E \omega_{\infty}-L \eta_{\infty}\right)\right.} \\
& \left.-\left(E \omega^{c}-L \eta^{c}\right)\right] \\
\breve{x}_{\infty}^{c}= & \mathbf{0} \\
\xi_{\infty}^{c}= & {[I-(A-L C)]^{-1}\left(E \omega^{c}-L \eta^{c}\right), } \\
\left\|\tilde{H}_{\infty_{i}}\right\|_{1}= & \left\|H_{\infty_{i}}^{\tilde{x}}\right\|_{1} \leq\left\|\breve{H}_{\infty_{i}}\right\|_{1},
\end{aligned}
$$

where $i$ represents the $i$-th row of a matrix and $\omega_{\infty} \in W$ and $\eta_{\infty} \in V$.

\footnotetext{
${ }^{1} x_{k}-\hat{x}_{k}$ is different from $x_{k}-\hat{x}_{k}$ in (12). The former corresponds to interval observers while the latter is from invariant sets. In this paper, $\breve{x}_{k}$ and $\tilde{x}_{k}$ are used to distinguish them.
} 
According to (26), the centers of $\tilde{X}_{\infty}$ and $\Phi_{\infty}^{\tilde{x}}$ have the following mathematical relationship:

$$
\tilde{x}_{\infty}^{c}+\xi_{\infty}^{c}=[I-(A-L C)]^{-1}\left(E \omega_{\infty}-L \eta_{\infty}\right),
$$

where $\tilde{x}_{\infty}^{c}+\xi_{\infty}^{c}$ are bounded. It is seen that in (26d) the size $^{2}$ of $\tilde{X}_{\infty}$ and $\Phi_{\infty}^{\tilde{x}}$ are the same and both are smaller than that of $\breve{X}_{\infty}$. Considering (26a), (26b) and (26d), $\tilde{X}_{\infty}$ has the same size but generally different centers with $\Phi_{\infty}^{\tilde{x}}$.

2) Relationship based on Residuals: In the interval observer-based FD, residual zonotopes defined in (9) can be rewritten as

$$
R_{k}^{i o}=C \tilde{X}_{k} \oplus\left\{\eta_{k}\right\} \oplus(-V)
$$

where $R_{k}^{i o}$ are always bounded by $\breve{R}_{k}^{i o}$ in (23), as long as $R_{0}^{i o} \subseteq \breve{R}_{0}^{i o}$. According to (27), the center $r_{k}^{i o, c}$ and segment matrix $H_{k}^{i o}$ of $R_{k}^{i o}$ have the following expressions:

$$
\begin{aligned}
r_{k}^{i o, c} & =C \tilde{x}_{k}^{c}+\left(\eta_{k}-\eta^{c}\right), \\
H_{k}^{i o} & =\left[\begin{array}{ll}
C \hat{H}_{k}^{x} & H_{\bar{\eta}}
\end{array}\right] .
\end{aligned}
$$

In the invariant set-based FD, substituting (24) and (25) into (14), the healthy residual set $R_{j}^{i s}$ is obtained as

$$
R_{j}^{i s}=C \Phi_{j}^{\tilde{x}} \oplus V
$$

where $j$ denotes the number of iterative steps indicated in Proposition 2.1 instead of the time instant. Similarly, the center $r_{j}^{i s, c}$ and segment matrix $H_{j}^{i s}$ of $R_{j}^{i s}$ are derived as

$$
\begin{aligned}
r_{j}^{i s, c} & =C \xi_{j}^{c}+\eta^{c}, \\
H_{j}^{i s} & =\left[\begin{array}{ll}
C H_{j}^{\tilde{x}} & H_{\bar{\eta}}
\end{array}\right] .
\end{aligned}
$$

According to (26), (28b) and (30b), as $k$ and $j$ tend to infinity, the size of $R_{k}^{i o}$ converges to that of the smallest residual set $R_{\infty}^{i s}$ corresponding to the mRPI set $\Phi_{\infty}^{\tilde{x}}$. The centers of $R_{\infty}^{i o}$ and $R_{\infty}^{i s}$ are generally different but have a mathematical relationship:

$$
r_{\infty}^{i o, c}+r_{\infty}^{i s, c}=C\left[I_{n}-(A-L C)\right]^{-1}\left(E \omega_{\infty}-L \eta_{\infty}\right)+\eta_{\infty}
$$

where it is known that $r_{\infty}^{i o, c}+r_{\infty}^{i s, c}$ is bounded.

This implies that, as $k$ tends to infinity, $R_{\infty}^{i o}$ will be a set that has the same size but generally different center (as indicated by (28) and (30)) with $R_{\infty}^{i s}$.

In the previous section, it is shown that residual zonotopes predicted by the interval observer have bounding zonotopes, i.e., $R_{k}^{i o} \subseteq \breve{R}_{k}^{i o}$. However, one can not assure that at infinity $R_{\infty}^{i s}$ is bounded by $\breve{R}_{\infty}^{i o}$. But, according to (26a) and (26b), a condition such that $R_{\infty}^{i s} \subseteq \breve{R}_{\infty}^{i o}$ is given as

$$
2\left(E \omega_{c}-L \eta_{c}\right) \in E W \oplus L(-V) .
$$

\footnotetext{
${ }^{2}$ In this paper, the size of a zonotope corresponds to the width of its interval hull.
}

\section{Summarizing the Discussions}

1) Summarizing the Relationship: Based on the residual form (9) and (12), the mathematical relationship between both FD approaches is briefly summarized as follows.

- The FD principle of both approaches is similar. In the invariant set-based approach, the healthy invariant set is fixed and determined offline but the residual is real-time obtained, while in the interval observer-based approach $\mathbf{0}$ is fixed but residual zonotopes are computed online.

- As $k$ tends to infinity, the size of residual zonotopes predicted by the interval observer converges to that of the smallest healthy residual set $R_{\infty}^{i s}$.

- The center of $R_{\infty}^{i o}$ has a mathematical relationship with that of $R_{\infty}^{i s}$.

- $R_{k}^{i o}$ is always bounded by $\breve{R}_{k}^{i o}$ and, under the condition (31), $R_{\infty}^{i s}$ is also bounded by $\breve{R}_{\infty}^{i o}$.

2) Faults Covered in the Study: Both FD approaches detect faults by testing the mismatch between the current and modelled system behavior.

Thus, once interval vectors predicted by the nominal interval observer do not include $\mathbf{0}$ or the residual exits the healthy residual set, it is considered that a fault occurred, which means that the aforementioned discussions are generally suitable for all detectable faults by both approaches.

Note that the established relationship can also be extended to the case of the faulty functioning, as long as both the interval observer and the invariant set are designed and computed according to the same faulty system model.

\section{Comparison Of The Two Methods}

\section{A. Comparison of Computational Aspects}

The computational burden of the interval observer-based approach is mainly from algorithms to compute intervals and the type of containment sets to propagate the effect of uncertainties on the system. In [10], interval observer algorithms are classified into region-based and trajectory-based algorithms. Generally, the former have lower computational burden than the latter and different types of containment sets require different computational efforts. Besides, the interval observer-based approach predicts state and output sets on line, which massively increases the computational burden.

In the invariant set-based approach, since the key invariant set is computed offline, the computational complexity of invariant set does not play a decisive role in the approach. During the runtime of the invariant set-based approach, its computational cost reduces to simple on-line set membership tests: check whether the residual exits its fixed healthy invariant set. Thus, comparatively, the invariant set-based approach has much lower computational burden.

\section{B. Comparison of Conservativeness}

Every type of sets used to enclose states or outputs inevitably results in a different degree of conservativeness. Besides, the interval observer algorithms also increase its conservativeness. As reported in [10], the absolute algorithms are generally less conservative than the relative algorithms. 
For the invariant set-based approach, the conservativeness is mainly from the size of invariant sets. According to [8], the invariant sets for on-line FD can approximate the mRPI set in arbitrarily expected precision. If a sufficiently small invariant set is obtained, the conservativeness could be reduced.

Additionally, in the invariant set-based approach, there are mainly two dynamic processes. The first one is the residual movement from the outside of the healthy invariant set to the inside while the second one is opposite. The former corresponds to the initial transient state and system recovery processes, while the latter corresponds to steady state FD and fault isolation (FI) processes. Since the interval observer-based FI has not been researched yet, this paper only focuses on the initial transient state and steady state FD, and omits system recovery and FI. In reality, since the invariant set is fixed and does not have adjustable flexibility, it is possible that the system initial condition is outside the healthy invariant set, which results in that the invariant setbased approach loses its effectiveness to detect faults during the initial transition state. However, theoretically, interval observers can reduce this conservativeness by arbitrarily assigning its initial set under the physical constraints of the system to contain the initial conditions.

It is shown that both approaches have their own advantages and disadvantages. The interval observers can provide system dynamic information during the whole process including the initial state, the transient and steady state, while invariant sets mainly reflect the system behaviors in steady state. Ideally, their joint use will be useful for mitigating their respective disadvantages and make use of their respective advantages.

\section{NUMERICAL EXAMPLE}

The following plant with sensor faults is used to illustrate the relationship between both approaches

$$
\begin{aligned}
x_{k+1} & =A x_{k}+B u_{k}+w_{k}, \\
y_{k} & =G_{i} C x_{k}+\eta_{k},
\end{aligned}
$$

where $G_{i}$ is a diagonal matrix to model the $i$-th mode $(i \in$ $\{0,1\}), G_{0}$ is the identity matrix modeling the healthy sensor situation and $G_{1}$ models the fault.

An interval observer designed as (7) is used to monitor the system. The residual and the residual zonotopes for the two approaches are defined as (9) and (12), respectively. The parameters of the illustrative example are given as

- model parameters: $A=\left[\begin{array}{cc}0.8667 & -1.2343 \\ 0.01 & 1\end{array}\right]$,

$$
B=\left[\begin{array}{cc}
0.01 & 1 \\
1 & 0.01
\end{array}\right], C=\left[\begin{array}{cc}
0.5 & 0 \\
0 & 1.5
\end{array}\right]
$$

- disturbances: $\bar{w}=\left[\begin{array}{ll}0.1 & 0.1\end{array}\right]^{T}, w^{c}=\left[\begin{array}{ll}0.1 & 0.1\end{array}\right]^{T}$,

- measurement noises: $\bar{\eta}=\left[\begin{array}{ll}0.1 & 0.1\end{array}\right]^{T}, \eta^{c}=\left[\begin{array}{ll}0.5 & 0.5\end{array}\right]^{T}$,

- observer gain and fault magnitude:

$$
L=\left[\begin{array}{cc}
0.5334 & -0.8229 \\
0.02 & 0.2
\end{array}\right], G_{1}=\left[\begin{array}{cc}
0.95 & 0 \\
0 & 1
\end{array}\right],
$$

- the control inputs, including two same components, are sinusoidal signals with offset $u^{c}=\left[\begin{array}{ll}2 & 2\end{array}\right]^{T}$ and magnitude $H_{u}=\left[\begin{array}{ll}0.2 & 0.2\end{array}\right]^{T}$,

- initial conditions:

$$
x_{0}=\left[\begin{array}{l}
0 \\
0
\end{array}\right], \hat{x}_{0}^{c}=\left[\begin{array}{l}
0.1 \\
0.1
\end{array}\right], \hat{H}_{0}^{x}=\left[\begin{array}{lll}
2 & 0 & 2 \\
0 & 2 & 2
\end{array}\right] .
$$

According to Theorem 2.1 and Proposition 2.1, one can compute an initial invariant set and then iterate the initial invariant set thirty steps to obtain a RPI approximation to sufficiently approach the mRPI set for the invariant set-based approach. The interval hull of the thirty-step healthy residual set is computed offline as $\square R_{30}^{i s}=([0.4114,1.2005],[-0.26,1.16])$, whose size is $(0.7891,1.42)$. In the sequel, this healthy residual set is used to illustrate the established relationships.

\section{A. Relationships in Terms of Set Sizes}

The relationship between residual zonotopes and the healthy residual set is shown in Figure 1. It is seen that residual zonotopes do not converge to the healthy residual set but their size converges to that of the healthy residual set. In the figure, after twenty-step on-line prediction, the size of residual zonotopes reaches $(0.7891,1.42)$ consistent with that of the healthy residual set.
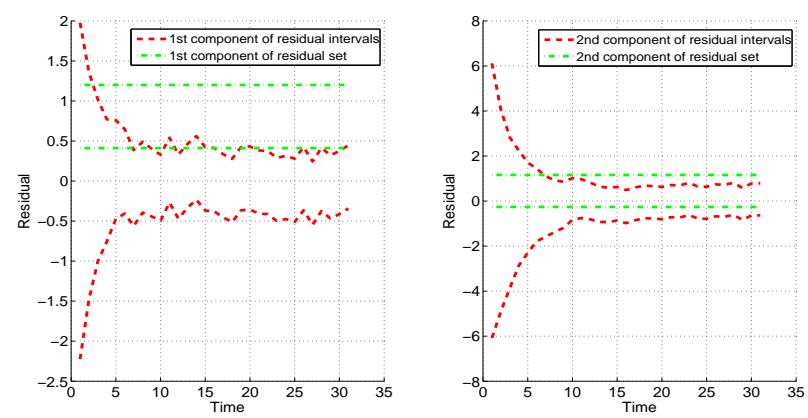

Fig. 1. Relationships in terms of set sizes

\section{B. Relationships in Terms of Bounds}

In Figure 2, the relationship among the healthy residual set, residual zonotopes and their bounds are shown. It is shown that residual zonotopes are always bounded by their bounds, while the healthy residual set is bounded by the bounds only under the conditions (31). Note that the center of the noise set for this simulation is $\eta^{c}=\left[\begin{array}{ll}0.1 & 0.1\end{array}\right]^{T}$.

\section{Relationships in Transient and Steady FD}

In Figure 3, the two approaches are used to detect the same faults during the initial transient and steady states, respectively. In Figure 3(a), the fault occurs at time instant $k=3$ and in Figure 3(b) the fault occurs at time instant $k=30$. It is seen that interval observers can detect the faults during both the initial transient and steady states while invariant set-based approach can only detect the fault in steady state, which shows the advantage of the interval 
observer in transient state FD and the similarity of the two approaches in steady state FD. In Figure 3 the center of the noise set and the observer gain are $\eta^{c}=\left[\begin{array}{ll}0.1 & 0.1\end{array}\right]^{T}$ and $L=\left[\begin{array}{cc}0.1334 & -0.8229 \\ 0.02 & 0.0667\end{array}\right]$
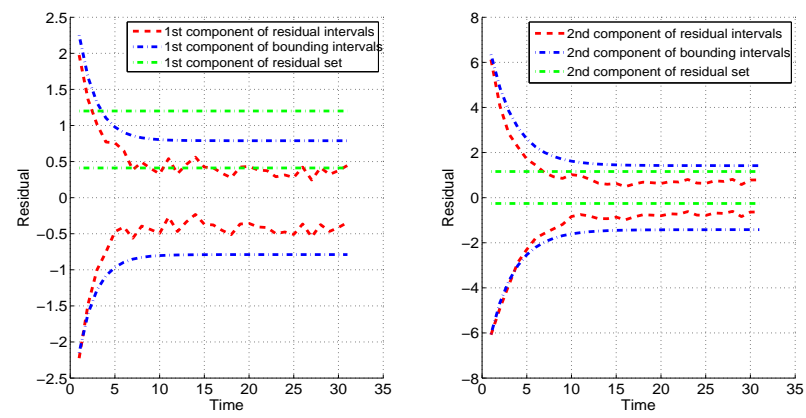

(a) Residual set outside bounding intervals
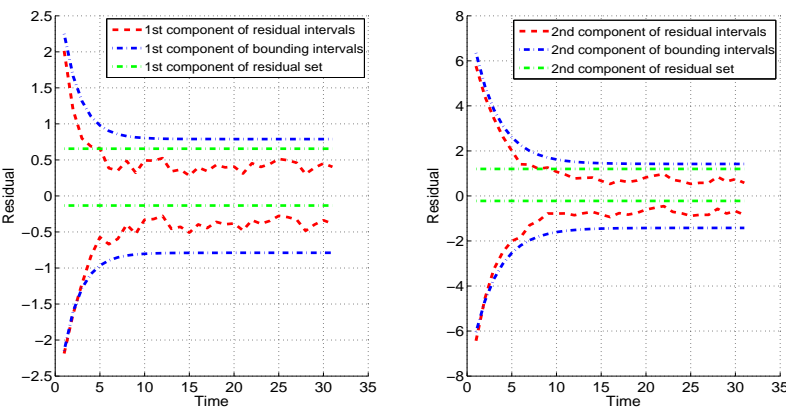

(b) Residual set inside bounding intervals

Fig. 2. Relationships in terms of bounds

\section{CONCLUSIONS}

This paper analyzes the interval observer-based and invariant set-based approaches, whose FD principle is similar. The former provides system information during the transient and steady state phases, but with more computational complexity. On the other hand, the latter focuses more on steady state of the system with lower computational burden. The future research is to explore the possibility of combining both approaches for more efficient FDI.

\section{ACKNOWLEDGMENT}

The work of F. Xu, V. Puig and C. Ocampo-Martinez has been supported by Spanish research projects WATMAN (CICYT DPI2009-13744) and CICYT SHERECS DPI-2011-26243 of the Science and Technology Ministry, by the DGR of Generalitat de Catalunya (SAC group Ref. 2009/SGR/1491), by European Comission through contract i-Sense (FP7-ICT-2009-6-270428), and by China Scholarship Council (File No.2011629170).

\section{REFERENCES}

[1] T. Alamo, J.M. Bravo, and E.F. Camacho. Guaranteed state estimation by zonotopes. In Decision and Control, 2003. Proceedings. 42nd IEEE Conference on, Maui, Hawaii, USA, December 2003.

[2] F. Blanchini and S. Miani. Set-theoretic Methods in Control. 2007.
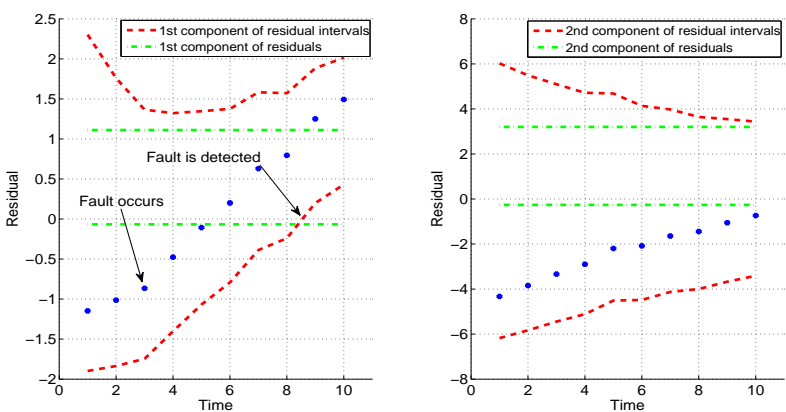

(a) FD in initial transient state
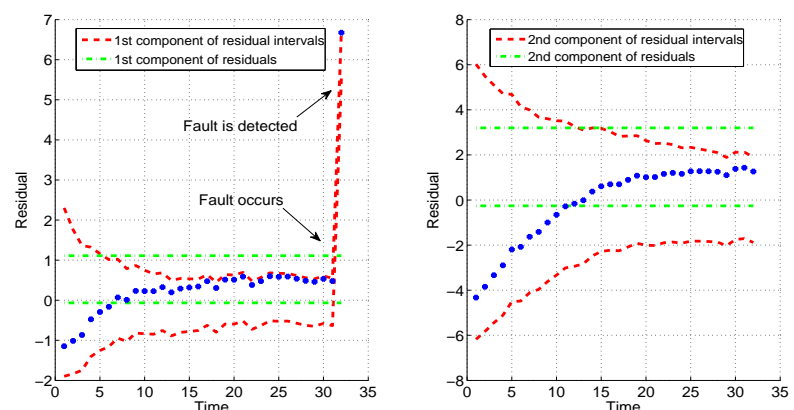

(b) FD in steady state

Fig. 3. Two approaches in FD

[3] C. Combastel. A state bounding observer based on zonotopes. In European Control Conference, Cambridge, UK, 2003.

[4] P. Guerra, V. Puig, and M. Witczak. Robust fault detection with unknown-input interval observers using zonotopes. In Proceedings of the 17th World Congress, The International Federation of Automatic Control, Coex, Seoul, South Korea, July 2008.

[5] F. Mazenc and O. Bernard. Interval observers for linear time-invariant systems with disturbances. Automatica, 47(1):140 - 147, 2011.

[6] J. Meseguer, V. Puig, and T. Escobet. Robust fault detection linear interval observers avoiding the wrapping effect. In Proceedings of the 17th World Congress, The International Federation of Automatic Control, Coex, Seoul, South Korea, July 2008.

[7] C. Ocampo-Martinez, J.A. De Doná, and M.M Seron. Actuator faulttolerant control based on set separation. International Journal of Adaptive Control and Signal Processing, 24(12):1070- 1090, 2010.

[8] S. Olaru, J.A. De Doná, M.M. Seron, and F. Stoican. Positive invariant sets for fault tolerant multisensor control schemes. International Journal of Control, 83(12):2622-2640, 2010.

[9] V. Puig, J. Quevedo, T. Escobet, and A. Stancu. Passive robust fault detection using linear interval observers. In IFAC Safe Process, Washington, USA, 2003.

[10] V. Puig, A. Stancu, and J. Quevedo. Observers for interval systems using set and trajectory-based approaches. In the 44th IEEE Conference on Decision and Control and 2005 European Control Conference, pages 6567 - 6572, Dec. 2005.

[11] S.V. Rakovic, E.C. Kerrigan, K.I. Kouramas, and D.Q. Mayne. Invariant approximations of the minimal robust positively invariant set. Automatic Control, IEEE Transactions on, 50(3):406 - 410, March 2005.

[12] M.M. Seron, X.W. Zhuo, J.A. De Doná, and J.J. Martínez. Multisensor switching control strategy with fault tolerance guarantees. Automatica, 44(1):88-97, 2008.

[13] F. Stoican. Fault tolerant control based on set-theoretic methods. $\mathrm{PhD}$ thesis, E3S-Supelec systems Science, Automatic Control Department, SUPÉLEC, France, October 2011. 